\title{
Cuestionario de Observación de la Conducta Bulímica (COBU): Desarrollo de un instrumento para cuidadores de pacientes con bulimia nerviosa
}

\author{
Idoia Dúo ${ }^{1}$, Ana R. Sepúlveda ${ }^{2}$, María León $^{2}$, Judith Pastor ${ }^{1}$ y M. Paz López ${ }^{1}$ \\ ${ }^{1}$ Centro ITEM, Madrid, España \\ ${ }^{2}$ Universidad Autónoma de Madrid, Madrid, España
}

\begin{abstract}
Resumen: El objetivo fue desarrollar y evaluar las propiedades psicométricas del Cuestionario de Observación de la Conducta Bulímica (COBU), sobre la sintomatología observada en la bulimia nerviosa $(\mathrm{BN})$ por los cuidadores. La muestra estaba constituida por 162 cuidadores de pacientes con $\mathrm{BN}(n=126)$, anorexia nerviosa $(n=26)$ y trastorno de atracón $(n=10)$. A través de un análisis exploratorio del COBU, se han aislado tres factores: Observación de Sobreingesta $(\alpha=0,77)$, Observación de Periodos de Restricción $(\alpha=0.76$ ) y Observación de Impulsividad $(\alpha=0.62)$. El cuestionario muestra buena validez convergente con la escala ABOS para cuidadores ( $\mathrm{rho}=0.58, p<0.01)$ y con el cuestionario BITE para pacientes (rho $=0.40, p<0.01$ ). El COBU discrimina entre diagnósticos clínicos, excepto la subescala de Observación de Impulsividad. Se recomienda su uso en evaluación familiar para ayudarles a reconocer y valorar los cambios en la sintomatología.
\end{abstract}

Palabras clave: Bulimia nerviosa; desarrollo; cuestionario; validez; cuidadores.

\section{Bulimic Behaviour Observation Questionnaire (COBU): \\ Developing an instrument for caregivers of patients with bulimia nervosa}

\begin{abstract}
The aim was to develop and evaluate the psychometric properties of the Bulimic Behaviour Observation Questionnaire (COBU), related to the symptoms observed in bulimia nervosa $(\mathrm{BN})$ by caregivers. The sample consisted of 162 caregivers of patients with BN ( $\mathrm{n}=126)$, anorexia nervosa (AN) $(n=26)$ and binge eating disorder $(n=10)$. Exploratory analysis of the COBU revealed three factors: Overeating observation $(\alpha=0.77)$, Restriction Interval Observation $(\alpha=0.76)$ and Impulsivity Observation $(\alpha=0.62)$. The questionnaire showed good convergent validity with the ABOS scale for carers $($ rho $=0.58), p<.01)$ and the BITE scale for patients (rho $=0.40, p<0.01$ ). The questionnaire discriminates between clinical diagnoses, except for the Impulsivity Observation subscale. It is recommended for use in the assessment by the family and to assist in recognizing and assessing changes in the symptoms.
\end{abstract}

Keywords: Bulimia nervosa; development; questionnaire; validity; caregivers.

\section{Introducción}

Según la reciente versión del Manual Diagnóstico y Estadístico de los Trastornos Mentales (DSM-5, 2013), los trastornos del comportamiento alimentario (TCA) se dividen en tres trastornos principales: anorexia nerviosa
(AN), bulimia nerviosa (BN) y trastorno de atracón (TA). El TA anteriormente pertenecía a un tercer subtipo denominado trastornos de la conducta alimentaria no especificado (TCA-NE), esta categoría era utilizada para codificar los TCA que no cumplían criterios para ningún TCA específico, de AN o BN. Las características actua-
Recibido: 8 marzo 2014; aceptado: 18 septiembre 2014.

Correspondencia: Judith Pastor, Av. De Pío XII, 57 Portal B, Bajo Izq. 28016 Madrid. Correo info@centroitem.com. A. R. Sepúlveda, Facultad de Psicología, Universidad Autónoma de Madrid. Campus de Cantoblanco.28049. Madrid. Correo-e: anarosa.sepulveda@uam.es
Agradecimientos: Agradecemos la valiosa colaboración de los pacientes con Trastorno de la Conducta Alimentaria y familiares que han participado en el estudio, así como al Hospital del Niño Jesús y muy especialmente al Dr. Morandé, jefe de psiquiatría del HNJS por estimular y facilitar este proyecto. La Dra. Sepúlveda disfruta de un contrato en el programa Ramón y Cajal del Ministerio de Ciencia e Innovación (RYC-2009-05092). 
les que los diferencian principalmente son las siguientes: las jóvenes con AN rechazan mantener un peso corporal en los valores mínimos normales, y utilizan la conducta de la restricción extrema para conseguir este fin; mientras que las jóvenes con BN presentan episodios recurrentes de voracidad, con una ingesta calórica enorme y una dificultad en parar la ingesta, seguidos de conductas compensatorias inapropiadas, como el uso del vómito autoprovocado, el abuso de laxantes, el ayuno y/o el ejercicio físico excesivo. El trastorno por atracón, se distingue por la presencia de episodios de sobreingesta, pero sin conductas compensatorias inapropiadas, por lo que suelen cursar con sobrepeso u obesidad. Una característica común de los tres diagnósticos es que la evaluación que hace la persona afectada sobre sí misma está influida exageradamente por la figura y el peso corporal (DSM-5, 2013). En cuanto a la etiología de esta patología, se considera que es multicausal, es decir, tanto factores biológicos como psicológicos y sociales intervienen en el origen y el mantenimiento de esta patología (Toro, Guerrero, Sentis, Castro y Puertolas, 2008).

Respecto a la prevalencia de los TCA, se estima que el $0.9 \%$ de los hombres y el $3.69 \%$ de las mujeres lo padecen. Cabe destacar que, de todas las personas que están diagnosticadas con un TCA, únicamente están recibiendo tratamiento un $43 \%$ de las que padecen $\mathrm{AN}$, un $12.5 \%$ de las que padecen $\mathrm{BN}$ y un $14.8 \%$ de las que padecen un TCA-NE (Oliva, Gandarillas, Sonego, Díez-Gañán y Ordobás, 2012). En relación a la evolución de estos trastornos, según los datos publicados en la Guía Práctica Clínica sobre los TCA (2009) en el caso de la AN, el 50\% de los casos evoluciona a remisión completa, la remisión parcial se observa entre el 20\%-30\% de los casos y entre el 10\%-20\% evolucionan hacia una cronicidad. En la BN, en estudios de seguimiento a seis años, el $60 \%$ se consideraban de buen pronóstico, el $30 \%$ de recuperación parcial y un $10 \%$ de mal pronóstico. En el caso de TA, en estudios de seguimiento a 5 años, el 70\% presentan un buen pronóstico, el 18\%-20\% tienen una remisión parcial y el 10\% mantenían el diagnóstico.

La necesidad de incorporar a la familia en el tratamiento de adolescentes y jóvenes adultos queda avalada por los resultados de distintas investigaciones en breve descritas. En concreto, el tratamiento basado en la familia (FBT) fue desarrollado en el Hospital Maudsley en Inglaterra. Este modelo terapéutico examina el impacto que la enfermedad de la paciente ocasiona en la familia, y cómo las respuestas de la propia familia pueden limitar la recuperación de la paciente. Diversos estudios muestran la comparación de este tratamiento (FBT) versus al tratamiento cognitivo-conductual individual, en población adolescente con AN y BN (Eisler, Dare, Russell, Szmukler, Le Grange y Dodge, 1997; Russell, Szmukler, Dare y Eisler, 1987). Los resultados se han dirigido inicialmente a pacientes con $\mathrm{AN}$, y se centraron exclusivamente en adolescentes (Eisler, Dare, Hodes, Russell, Dodge y Le Grange, 2000; Eisler, Simic, Russell y Dare, 2007). Estos estudios han demostrado la eficacia de este tratamiento familiar, donde aproximadamente dos tercios de los pacientes adolescentes con AN se recuperan. Este enfoque fue difundido, probado en otros países y adaptado para una aproximación transdiagnóstica del problema. El modelo transdiagnóstico postula una continuidad de las categorías diagnósticas, donde el curso de la evolución de la sintomatología de la paciente cambia en el tiempo (Fairburn, Cooper y Shafran, 2003), lo que parece claro, es que la patología afecta a la familia y a su entorno, y pueden permitir la progresión y mantenimiento del TCA (Loeb, Lock, Greif y Le Grange, 2012). En estos momentos, se han desarrollado manuales de intervención en FBT para pacientes con AN (Lock, Le Grange, Agras y Dare, 2001), y para pacientes con BN (Le Grange y Lock, 2007). Los resultados muestran que los adolescentes con BN tratados por FBT mostraban mayores tasas de recuperación de los síntomas bulímicos, al final de tratamiento y en el seguimiento, en comparación con aquellos que recibieron la terapia de apoyo individual (Le Grange, Crosby, Rathouz y Leventhal, 2007; Lock, Agras, Bryson y Kraemer, 2005). La intervención cognitivo-conductual para pacientes adolescentes y sus padres en el tratamiento de la BN es más eficaz que el tratamiento individual en cuanto a los abandonos y las recaídas (Castro, Larroy y Gómez, 2010).

Por tanto, la implicación de los familiares en el tratamiento tiene un importante valor terapéutico, desde el momento de la evaluación de la paciente. La negación y/o minimización de los síntomas es inherente a los TCA. Las personas con TCA pueden minimizar síntomas o esconder conductas. Además dado que son los padres quienes detectan los primeros síntomas del TCA en el adolescente, esta información es de gran importancia para su detección precoz y así disminuir la comorbilidad y mortalidad en adolescentes con TCA. Por ello la Guía del Lucile Salter Children's Hospital de Stanford para adolescentes con TCA recomienda incluir a la familia no sólo en el proceso de tratamiento sino también en la evaluación inicial (Rome et al., 2003). En este mismo sentido, la Guía Práctica para TCA de la APA (Yager, Anderson y Devlin, 2000) señala la importancia de involucrar a la familia en la evaluación y el tratamiento de niños y adolescentes con esta patología, y recomienda incluirlos desde las primeras etapas de diagnóstico. 
Se concluye que es de enorme importancia diseñar e incorporar en la intervención de los TCA instrumentos de medida válidos para familiares que ayuden en el proceso de evaluación. No obstante, el estudio y desarrollo de cuestionarios de TCA para familiares todavía está en vías de desarrollo. La revisión de la literatura en la última década muestra que se han desarrollado varios instrumentos, como por ejemplo, a) el Cuestionario de Necesidades de los Cuidadores (CaNAM; Carers'Needs Assessment Measure; Haigh y Treasure, 2003) es un instrumento que evalúa las necesidades (ej. información sobre los trastornos alimentarios, tipos de tratamiento, organizaciones de apoyo), no existe validación española; b) La Escala del Impacto de los Síntomas de los Trastornos Alimentarios (EDSIS; Eating Disorders Symptom Impact Scale; Sepúlveda et al., 2008) evalúa el impacto específico de la sintomatología alimentaria como el aislamiento social de la paciente o las conductas disfuncionales en la familia. Resultó sensible a las mejoras en las habilidades de afrontamiento de los familiares tras recibir una intervención psicoeducativa. Se ha validado en una muestra de familiares españoles con apropiadas medidas psicométricas (Carral et al., 2013); c) La escala de Acomodación e Incompetencia con los Síntoma Alimentarios (AESED; Accommodation and Enabling Scale for Eating Disorders; Sepúlveda, Kyriacou y Treasure, 2009) evalúa la adaptación a los síntomas por parte de los familiares, y la respuesta de incompetencia a la patología, como por ejemplo, ayudan en los rituales de las comidas, o modifican sus rutinas propias para evitar conflictos. Recientemente validada en castellano (Quiles et al., 2015); por último destaca dos instrumentos de auto-informe que miden el clima familiar y la emoción expresada, principalmente se han desarrollado en base al ambiente familiar en esquizofrenia, d) la escala del Nivel de Emoción Expresada (LEE; Level of Expressed Emotion; Cole y Kazarian, 1988). Esta escala cuenta con dos versiones, la emoción expresada desde el punto de vista del familiar y desde la perspectiva del paciente. Este instrumento se ha validado en castellano en una muestra de familias de TCA, su versión se ha reducido a 45 ítems, con adecuadas propiedades psicométricas ( $\mathrm{Se}-$ púlveda et al., 2012) y el otro instrumento, e) el Cuestionario Familiar (FQ; Family Questionnaire; Wiedemann, Rayki, Feinstein y Hahlweg, 2002). Consta de dos subescalas, comentarios críticos y sobreimplicación emocional. Es una escala útil por su brevedad, tiene 20 ítems y puntos de corte para cribar. Sepúlveda et al., (2014) han validado recientemente en castellano este instrumento en cuidadores de TCA.

Respecto a los instrumentos de evaluación existentes que midan la sintomatología TCA a través de la obser- vación por parte de la familia, con el fin de buscar una mayor fiabilidad de la observación de la gravedad de los síntomas y actitudes ante la enfermedad, encontramos: a) la Escala de Observación de la Conducta Restrictiva (ABOS; Anorectic Behavior Observation Scale for parents; Vandereycken et al., 1992). Se utilizan para hacer un diagnóstico diferencial con BN. También se puede utilizar para detectar personas con sospecha de TCA en la etapa temprana. Consta de tres subescalas, que miden el comportamiento relacionada con el peso y/o comida y la negación del problema, el comportamiento bulímico y las conductas de hiperactividad. El cuestionario está validado recientemente (Martin et al., 2013); b) el Examen de Trastornos de la Conducta Alimentaria, versión padres (P-EDE; Loeb, 2008). Fue desarrollado para mejorar la identificación de casos TCA en niños y adolescentes mediante el empleo de múltiples informantes (padres). El P- EDE se basa en la entrevista clínica EDE16 (Fairburn, Cooper y O'Connor, 2008). Estudios recientes ponen de relieve que la información de los padres y el clínico son importantes en la evaluación de los niños y adolescentes con AN al usar P-EDE, pero parece ser menos crítico para evaluar personas con BN. Existe una versión del P-EDE en formato cuestionario (P-EDEQ; Loeb, 2007), basada en el EDEQ, y por último, c) el Cuestionario de Patrones de Alimentación y Peso, versión padres (QEWP-P; Johnson, Grieve, Adams y Sandy, 1999). El QEWP fue desarrollado por Spitzer y colaboradores en el año de 1992. Se utiliza para identificar personas con trastorno por atracón. Este trastorno puede aparecer en niños y adolescentes, por lo que Johnson y colaboradores (1999) adaptaron una versión para adolescentes (QEWP-A) y otra para padres (QEWP-P). Los estudios con este cuestionario sugieren que los padres tienen un sentido limitado de lo que ocurre en términos de los hábitos privados de alimentación, característico de los atracones o de la BN (Tanofsky-Kraff, Yanovski \& Yanovski, 2005), no existe validación en castellano.

Actualmente, no se tiene constancia de la existencia de un cuestionario de observación para cuidadores, específico para la $\mathrm{BN}$, con el fin de extraer información que ayude a la detección y gravedad de esta patología. Es importante aclarar que vamos a utilizar a partir de ahora el término cuidador, definido como aquella persona que cuida y pasa el mayor número de horas con la paciente en el momento de la evaluación (Berbel et al., 2010).

\section{Objetivos del estudio}

El objetivo principal del estudio, fue el desarrollo y la evaluación de las propiedades psicométricas de un 
cuestionario de observación de sintomatología bulímica para familiares (sobre la sintomatología de Bulimia Nerviosa presente en el familiar afectado). Este instrumento se ha denominado "Cuestionario de Observación de la Conducta Bulímica” (COBU). Los objetivos específicos fueron los siguientes: a) Desarrollar ítems que evalúen la sintomatología en $\mathrm{BN}$ a través de expertos clínicos y un grupo de familias, b) examinar a través de una análisis exploratorio la estructura factorial del cuestionario, c) estudiar la consistencia interna del instrumento (alfa de Cronbach) y e) estudiar la validez convergente del cuestionario COBU con otros instrumentos que evalúan el TCA y su sensibilidad para cribar diagnóstico clínico.

Respecto a la validez convergente, se espera que el instrumento $\mathrm{COBU}$ correlacione positivamente y de forma elevada con el cuestionario para padres ABOS. Se espera que correlacione de forma moderada con los dos cuestionarios completados por las pacientes, el BITE y las cuatro subescalas del EDI-2 (Obsesión por la Delgadez, Bulimia, Insatisfacción corporal e Impulsividad). Se espera que el COBU pueda discriminar pacientes con $\mathrm{BN}$ de pacientes con $\mathrm{AN}$, y ser similar a pacientes con TA, excepto en las conductas relacionadas con la impulsividad.

\section{Método}

\section{Desarrollo del cuestionario}

Tras la revisión de la literatura inicial, y de los instrumentos desarrollados para familiares, se ha utilizado como referencia el cuestionario ABOS (especifico de la AN) (Vandereycken et al., 1992), para desarrollar ítems de forma parecida para medir la BN, y el Test de Bulimia de Edinburgo (BITE, Bulimic Investigatory Test Edinburgh; Herdenson y Freeman, 1987) (dirigido a la evaluación de pacientes con $\mathrm{BN}$ ). Los ítems de conductas compensatorias se recogieron con el mismo formato de respuesta de este cuestionario. Un panel de tres psicólogas clínicas especializadas en TCA (co-autoras ID, JP, y MPL), elaboraron aproximadamente 50 ítems que evalúan distintos aspectos psicopatológicos de la bulimia nerviosa (p.ej., restricción alimentaria, obsesión por la delgadez, impulsividad, conductas compensatorias, entre otras) que usualmente se recogen en las entrevistas clínicas. Posteriormente, se creó un grupo de familiares de pacientes de BN, para que valoraran la escalas, y así se descartaron algunos de los ítems por ser poco claros o por no ser muy representativos.

En el cuestionario COBU se recogen cuestiones relacionadas con la restricción alimentaria, impulsividad hacia la comida, conductas purgativas (vómitos, laxantes, ejercicio compulsivo, etc.), la imagen corporal, el consumo de sustancias y otros aspectos relacionados con las relaciones sociales o entre iguales (ver Anexo I).

Finalmente, el instrumento inicial constaba de un total de 30 ítems, donde, 28 de ellos se presentan con tres opciones de respuesta: "SI" ( 2 puntos), "NO" (0 puntos) o "?" (1 punto) y dos de ellos (ítems 5 y 9) tienen un formato de respuesta tipo Likert con 8 opciones de respuesta y se califican de 0 a $7(0=$ nunca; $1=$ no lo sabe; $2=$ ocasionalmente; $3=1 \mathrm{vez} / \mathrm{semana} ; 4=2$ ó 3 veces $/$ semana; $5=$ diariamente; $6=2$ ó 3 veces $/$ día; $7=3$ o más veces/día). Cabe destacar, que el ítem 5 incluye cinco conductas compensatorias que el cuidador debe haber observado en la paciente y puntuar en dicha escala.

El cuestionario COBU presenta una estructura similar al cuestionario BITE con escalas de síntomas y gravedad. Los ítems correspondientes a la subescala de Síntomas tienen formato de respuesta "SI", "NO" o "?"; mientras que los ítems pertenecientes a la subescala de Gravedad tienen formato de respuesta tipo Likert.

\section{Participantes}

La muestra estuvo constituida por un total de 162 cuidadores de pacientes con trastornos de la conducta alimentaria (TCA), pudiendo ser la madre, el padre, la pareja o una amiga de la paciente. E1 70.6\% de los cuestionarios $(n=120)$ fueron administrados en el Hospital Niño Jesús (HNJS) de la Comunidad de Madrid y el 29.4\% $(n=50)$ restante en el Centro ITEM, un centro privado especializado en TCA situado en la Comunidad de Madrid. Un total de ocho personas fueron excluidas por no completar la batería de cuestionarios. Las pacientes fueron evaluadas por entrevista clínica siguiendo el DSM-IV-TR (APA, 2002). Las tres psicólogas clínicas (I.D., J.P., y MP.L.) recogieron la muestra tanto en el centro hospitalario HNJS como en el Centro privado, utilizando el mismo protocolo clínico, supervisada por el jefe de Psiquiatría (Dr. GM). El 77.8\% de la muestra son cuidadores de pacientes con $\mathrm{BN}(n=126)$, el $6.2 \%$ son cuidadores de pacientes con TA $(n=10)$ y el $16 \%$ son cuidadores de pacientes con AN $(n=26)$ (ver Tabla 1$)$. La submuestra de AN no se ha incluido para el análisis factorial; sin embargo, se ha utilizado para calcular la sensibilidad de la respuesta al cuestionario y para valorar el diagnóstico diferencial. En la Tabla 1 se presentan los datos sociodemográficos de los cuidadores y de los pacientes con TCA. También muestra los datos clínicos de los pacientes con TCA.

En la Tabla 2 se indica información sobre la edad, el índice de masa corporal (IMC), el tiempo en tratamiento y la realización de la recogida de datos según el diagnós- 
Tabla 1. Variables sociodemográficas y clínicas en los grupos de cuidadores y pacientes con trastornos de la conducta alimentaria

\begin{tabular}{|c|c|c|c|}
\hline \multicolumn{2}{|l|}{ Características de los cuidadores } & \multirow{3}{*}{$\begin{array}{c}N \\
152\end{array}$} & \multirow{3}{*}{$\begin{array}{c}\text { Media }(\mathrm{DT}) / \text { Porcentaje } \\
47.04(D T=7.3) \\
\text { Rango }=20-66\end{array}$} \\
\hline Edad* & & & \\
\hline & & & \\
\hline \multirow[t]{5}{*}{ Tipo de cuidador } & Madre & 141 & $87.0 \%$ \\
\hline & Padre & 12 & $7.4 \%$ \\
\hline & Hermanos/as & 3 & $1.9 \%$ \\
\hline & Pareja/novio & 4 & $2.5 \%$ \\
\hline & Amigos/as & 2 & $1.2 \%$ \\
\hline \multirow[t]{3}{*}{ Nivel Educativo* } & Hasta Graduado Escolar & 43 & $28.5 \%$ \\
\hline & Bachillerato o superior & 42 & $27.8 \%$ \\
\hline & Universidad/diploma & 66 & $43.7 \%$ \\
\hline \multirow[t]{3}{*}{ Estatus Marital* } & Casados/Vive en pareja & 124 & $78.5 \%$ \\
\hline & Divorciado/separado & 28 & $17.7 \%$ \\
\hline & Soltero & 6 & $3.8 \%$ \\
\hline \multirow[t]{3}{*}{ Situación Laboral* } & Tiempo completo/parcial & 114 & $73.1 \%$ \\
\hline & Desempleado/Ama de casa & 36 & $23.1 \%$ \\
\hline & Estudiante & 6 & $3.8 \%$ \\
\hline \multirow[t]{2}{*}{ Periodo de recogida } & Inicio de Tratamiento & 99 & $61.1 \%$ \\
\hline & Seguimiento & 63 & $38.9 \%$ \\
\hline \multicolumn{4}{|c|}{ Características de las Pacientes $(n=162)$} \\
\hline \multirow[t]{2}{*}{ Edad* } & & 160 & $17.73(D T=3.7)$ \\
\hline & & & Rango $=12-35$ \\
\hline \multirow[t]{2}{*}{ Índice de Masa Corporal* } & & 155 & $21.9(D T=3.7)$ \\
\hline & & & Rango $=15.3-40.4$ \\
\hline \multirow[t]{2}{*}{ Tiempo de tratamiento (meses)* } & & 141 & $10(D T=17.3)$ \\
\hline & & & Rango $=1-132$ \\
\hline \multirow[t]{2}{*}{ Género } & Mujer & 157 & $96.9 \%$ \\
\hline & Varón & 5 & $3.1 \%$ \\
\hline \multirow[t]{3}{*}{ Nivel Educativo* } & Primaria/Secundaria & 50 & $31.4 \%$ \\
\hline & Bachiller/FP & 83 & $52.2 \%$ \\
\hline & Universitario/Diploma & 26 & $16.4 \%$ \\
\hline \multirow[t]{3}{*}{ Estatus laboral } & Estudiante & 143 & $88.3 \%$ \\
\hline & Trabajo completo & 15 & $9.3 \%$ \\
\hline & Sin trabajo & 4 & $2.4 \%$ \\
\hline \multirow[t]{2}{*}{ Estatus Marital } & Casados/Convivencia & 3 & $1.9 \%$ \\
\hline & Soltera & 159 & $98.1 \%$ \\
\hline \multirow[t]{3}{*}{ Tipo de Tratamiento } & Ingreso & 1 & $0.6 \%$ \\
\hline & Hospital de Día & 11 & $8.0 \%$ \\
\hline & Ambulatorio & 150 & $91.4 \%$ \\
\hline \multirow[t]{6}{*}{ Diagnóstico según DSM-IV-R } & AN-R & 11 & $6.8 \%$ \\
\hline & AN-P & 15 & $9.3 \%$ \\
\hline & BN-P & 78 & $48.1 \%$ \\
\hline & BN-NP & 17 & $10.5 \%$ \\
\hline & TCA-NE subtipo BN-P & 31 & $19.1 \%$ \\
\hline & TCA-NE Subtipo TA + sobrepeso & 10 & $6.1 \%$ \\
\hline \multirow[t]{3}{*}{ Diagnósticos agrupados } & $\mathrm{AN}-\mathrm{R}+\mathrm{AN}-\mathrm{P}$ & 26 & $16 \%$ \\
\hline & $\mathrm{BN}-\mathrm{P}+\mathrm{BN}-\mathrm{NP}+\mathrm{TCA}-\mathrm{NE}$ & 126 & $77.8 \%$ \\
\hline & $\mathrm{TA}+$ sobrepeso & 10 & $6.2 \%$ \\
\hline
\end{tabular}

Nota. $\mathrm{AN}=$ anorexia nerviosa, $\mathrm{BN}=$ bulimia nerviosa, $\mathrm{TA}=$ trastorno de atracón, $\mathrm{TCA}=$ trastorno de la conducta alimentaria, $\mathrm{NE}=$ no especificado, $\mathrm{NP}=$ tipo no purgativo, $\mathrm{P}=$ tipo purgativo, $\mathrm{R}=$ tipo restrictivo. $*$ No se dispone de la información de todos los participantes $(N=162)$. 
Tabla 2. Diferencias en la distribución de medias y desviaciones típicas de variables clínicas según diagnósticos clínico

\begin{tabular}{|c|c|c|c|c|c|}
\hline Grupo clínico & $n$ & $\begin{array}{c}\text { Edad } \\
M(D T)\end{array}$ & $\operatorname{IMC}\left(\mathrm{kg} / \mathrm{m}^{2}\right)$ & $\begin{array}{c}\text { Tiempo } \\
\text { Tratamiento } \\
\text { (meses) } \\
M(D T)\end{array}$ & $\begin{array}{c}\text { Fase de recogida } \\
(\%)\end{array}$ \\
\hline $\mathrm{AN}$ & 26 & $17.5(3.7)$ & $18.9(2.0)$ & $8.8(14.8)$ & $61 \%$ evaluación \\
\hline $\mathrm{BN}$ & 126 & $17.8(3.8)$ & $21.9(2.8)$ & $10.5(18.3)$ & 64\% evaluación \\
\hline \multirow[t]{2}{*}{ TA } & 10 & $16.4(23)$ & $28.8(6.4)$ & $7.3(7.5)$ & $40 \%$ evaluación \\
\hline & & $F=0.58$ & $F=36 * * *$ & $F=0.20$ & $\chi^{2}=2.2$ \\
\hline
\end{tabular}

Nota. $\mathrm{ICM}=$ índice de masa corporal, $\mathrm{AN}=$ anorexia nerviosa, $\mathrm{BN}=$ bulimia nerviosa, $\mathrm{TA}=$ trastorno de atracón. $* * * p<.001$.

tico clínico agrupado, separando el TA según el DSM-5 (APA, 2013). Cabe destacar que tras realizar los análisis de varianza, la media de edad en los tres grupos de TCA (AN, BN y TCA-NE) es similar ( $p>0.05)$; el IMC difiere entre los distintos tipos diagnósticos, siendo la mayor diferencia encontrada entre los pacientes con AN y los pacientes con TA $(p<0.01)$, como cabría esperar. No hay diferencias significativas para el tiempo en tratamiento de cada paciente o respecto al momento de la recogida de datos (la mayoría en la fase de evaluación inicial).

\section{Instrumentos de Evaluación}

(a) Evaluación de los cuidadores

Datos sociodemográficos y clínicos. Los datos que se han recogido de los cuidadores son los siguientes: la edad, el sexo, el nivel de estudios, la situación laboral, el estado civil, y el tipo de relación del observador.

Cuestionario de Observación de la Conducta Bulímica $(C O B U)$. Este instrumento constaba de 30 ítems. El cuestionario presenta dos subescalas: Síntomas (28 ítems con formato de respuesta dicotómica) y Gravedad ( 2 ítems con formato de respuesta tipo likert). Se ha diseñado específicamente para cuidadores de pacientes con bulimia nerviosa (BN). Una mayor puntuación en las escalas, indica mayor gravedad de las conductas bulímicas del paciente.

La Escala de Observación de la Conducta Restrictiva (ABOS, Anorectic Behavior Observation Scale for parents; Vandereycken et al., 1992). Es una escala de observación de conductas y actitudes comunes de sintomatología en AN para ser completada por los padres. Está formado por 30 ítems, con tres posibilidades de respuesta: "SI" (2 puntos), "NO" (0 puntos) y "?" (1 punto). Su rango oscila entre 0 y 60 . La fiabilidad del instrumento original es de 0.95 . La fiabilidad en la validación española fue de 0.81 (Martin et al., 2013). Una mayor puntuación indica una mayor gravedad clínica de la paciente. (b) Evaluación de las pacientes

Datos sociodemográficos y clínicos. Los datos que se han recogido de las pacientes son los siguientes: el diagnóstico clínico, el tiempo de evolución, los tratamientos anteriores, el tratamiento actual, la edad, el sexo, el nivel de estudios, la situación laboral, el estado civil, el tipo de relación del observador, el peso $(\mathrm{kg})$ y la altura $(\mathrm{m})$ con una báscula y un tallímetro, y se calculó el IMC posteriormente.

Inventario de Trastornos de la Conducta Alimentaria (EDI-2, Eating Disorder Inventory-2; Garner, 1991). Se utilizó la versión española de Garner (1998).

El cuestionario consta de 91 ítems, con 6 alternativas de respuesta (de 1 (nunca) a 6 (siempre), divididos en 11 subescalas. La consistencia interna para las 11 escalas fluctúa entre 0.84 y 0.92 . La validación en muestra española indica también buenas propiedades psicométricas y su fiabilidad varía entre $\alpha=0.69$ y $\alpha=0.93$. Para este estudio, sólo hemos utilizado las siguientes 4 subescalas: la Obsesión por la Delgadez, la Bulimia, la Insatisfacción Corporal y la Impulsividad. Las altas puntuaciones indican una mayor patología en esa dimensión.

Test de Bulimia de Edimburgo (BITE, Bulimic Investigatory Test Edimburgh; Henderson y Freeman, 1987) es un cuestionario que evalúa la sintomatología bulímica. Está formado por 36 ítems con dos escalas, una escala de Síntomas y otra escala de Gravedad de esos síntomas. La fiabilidad del instrumento original tiene un valor de $\alpha=0.96$ para la escala de Síntomas y una fiabilidad de $\alpha=0.62$ para la escala de Gravedad. Rivas, Jimenez, y Bersabe (2004) han validado este cuestionario con una muestra de adolescentes españoles. La fiabilidad encontrada en la muestra española ha sido de $\alpha=0.82$ para la escala de Síntomas, y $\alpha=0.63$ para la escala de Severidad. A mayor puntuación en las escalas, mayor frecuencia de síntomas y mayor severidad. 


\section{Procedimiento}

El presente estudio consiste en una investigación de tipo transversal. La muestra se ha recogido durante aproximadamente cuatro años (2003-2006), por lo que la batería de cuestionarios se administró en la mayoría de las pacientes y sus familiares durante la fase de evaluación clínica de forma consecutiva, tanto en el Hospital Niño Jesús (HNJS) como en el centro ITEM. Esta batería formó parte del protocolo del hospital durante ese periodo, es decir, las pacientes y sus familiares (principalmente) son evaluados a través de la entrevista clínica por los psiquiatras y psicólogos de la Unidad de TCA, y se pedía que cumplimentarán una batería más amplia de cuestionarios, tanto a los padres como a las familias, donde estaban estos cuestionarios incluidos para el estudio. En el Hospital se recogen a la semana de realizar la entrevista para completar la evaluación clínica y en el Centro ITEM tras 1 ó 2 semanas. La duración de completar toda la batería para cada uno es de 1 hora aproximadamente.

\section{Análisis estadísticos}

Para el cálculo de la validez de constructo se utilizó el análisis factorial exploratorio mediante la extracción de componentes principales y ejes principales, aplicando el método de rotación varimax; se partió de peso mínimo aceptable de 0.35 , como criterio de saturación para ser un ítem seleccionado. La rotación varimax se asume como el método más adecuado, dado que se espera discriminar el máximo de factores que forman la escala. A su vez también se ha utilizado el método más conservador y por tanto más riguroso de extraer los factores por ejes principales y rotación varimax. Se calculó los estimadores de adecuación muestral de Kaiser-Meyer-Olkin (KMO; rango entre 0-1) y de significación estadística de Bartlett (si su valor es cercano a la unidad y son significativos $p<0.05$, indican que el análisis con reducción de variables es adecuado).

Para la prueba de normalidad de las escalas del COBU y el resto de la batería se utilizó el procedimiento Kolmogorov-Smirnov. Como no cumplía con el criterio de tener una distribución normal, el cálculo de la validez convergente entre el COBU total y el ABOS de los padres, y con las cuatro subescalas del EDI-2, Obsesión por la Delgadez, Bulimia, Insatisfacción corporal e Impulsividad y el BITE de la propias pacientes, se usó el coeficiente de correlación de Spearman. De igual modo, se utilizó el coeficiente de correlación de Spearman para conocer la intercorrelación entre los diferentes factores del cuestionario COBU. Cohen (1988) sugirió como guía para interpretar los resultados de las correlaccio- nes: igual o mayor que 0.50 es elevado, $0.49-0.30$ es moderado, $0.29-0.10$ es pequeño.

Para calcular la validez clínica del cuestionario se utilizó una submuestra de pacientes con AN y se calculó un ANOVA de un factor, aplicando la prueba post hoc de Bonferroni. Para el análisis de los datos se utilizó el programa estadístico SPSS 19.0. en su versión para Windows (SPSS, 2013). Los análisis estadísticos se han realizado con un nivel de significación de $p \leq 0.05$.

\section{Resultados}

\section{Análisis factorial}

Se realizaron análisis de componentes principales y rotación oblicua (varimax). La medida de adecuación muestral (KMO) fue 0.72, superando el valor recomendado de 0.60 y el estimador de esfericidad de Bartlett fue estadísticamente significativo $(p<0.01)$. La solución inicial encontrada contenía 11 factores con autovalores mayores que 1. Estos 11 factores explicaban un $64 \%$ de la varianza total, pero algunos de ellos incluían sólo uno o dos ítems y explicaban un porcentaje de varianza muy pequeño. Se ha utilizado la gráfica de sedimentación para valorar el número de factores a obtener. Los resultados mostraron tres factores claros y el resto se situaba entorno a 1. Posteriormente, se repitieron los análisis de componentes principales (CP), aplicando también de ejes principales (EP), pero esta vez forzando la extracción a tres componentes. Destaca el Ítem 18 “¿Considera que la máxima preocupación de su hija/o es la comida?", que satura en el Factor 1 y en el Factor 2, obteniendo una máxima puntuación en el Factor 2, a través del análisis de CP. Finalmente, se ha decidido asociarlo al Factor 1 tras los resultados del análisis de EP, donde sólo satura en el Factor 1. En la Tabla 3 se puede apreciar la matriz de componentes rotados final.

El cuestionario original contaba con 30 ítems. Sin embargo, tres ítems saturaron por debajo de 0.35 , y no han quedado incluidos en ningún factor. Como consecuencia, el cuestionario final consiste en 27 ítems (dos de ellos, ítems 5 y 9, tienen formato de respuesta tipo Likert, por lo tanto no aparecen en el análisis factorial).

\section{Corrección del cuestionario $C O B U$}

En la corrección del cuestionario COBU se deben tener en cuenta las dos subescalas. Por un lado, para hallar la puntuación en gravedad se suman las puntuaciones obtenidas en los 2 ítems 5 y 9 (tipo Likert). La puntuación en gravedad fluctuará entre 0 y 42. En la Tabla 3 puede observarse la distribución de frecuencias de las 
Tabla 3. Frecuencia de las conductas compensatorias y punto de corte de riesgo $\geq 5$

\begin{tabular}{lrrr}
\hline \multicolumn{1}{c}{$\begin{array}{c}\text { Conductas compensatorias } \\
\text { Items 5 y 9. Gravedad }\end{array}$} & $\begin{array}{c}\text { Nunca }-2-3 \text { veces/semana }(0-4) \\
\%(n)\end{array}$ & $\begin{array}{c}\text { Diariamente o más veces al día }(5-7) \\
\%(n)\end{array}$ & Media $(D T)$ \\
\hline 5a. Uso de píldoras adelgazantes & $100 \%(n=138)$ & $0 \%(0)$ & $0.13(0.4)$ \\
5b. Uso de diuréticos & $98.5 \%(n=131)$ & $1.5 \%(n=2)$ & $0.15(0.7)$ \\
5c. Uso de laxantes & $97 \%(n=128)$ & $3.0 \%(n=4)$ & $0.55(1.2)$ \\
5d. Vómito autoinducido & $77 \%(n=89)$ & $23 \%(n=27)$ & $2.40(2.2)$ \\
5e. Ejercicio compulsivo & $92 \%(n=125)$ & $8.0 \%(n=11)$ & $1.57(1.8)$ \\
9. Episodios de Atracones & $77 \%(n=22)$ & $23 \%(n=5)$ & $2.8(1.6)$ \\
\hline
\end{tabular}

conductas compensatorias evaluadas mediante estos ítems.

Por otro lado, para hallar la puntuación en Síntomas se deberán sumar las puntuaciones obtenidas en el resto de ítems. Se debe considerar que se han encontrado tres factores, ver Tabla 4. El primero de ellos se ha denominado "Observación de Sobreingesta", ya que los ítems que han saturado para este primer factor están relacionados con la desaparición de la comida, atracones, comer una mayor cantidad de comida de lo que se considera normal, rapidez a la hora de ingerir alimentos, entre otros. Se suma la puntuación obtenida en los ítems 11, $13,18,10,16,14,19,21,7$ y 8 . El segundo factor se ha denominado "Observación de Periodos de Restricción" debido a que los ítems que han saturado se relacionan con aspectos restrictivos como el ayuno, las dietas, la evitación de determinados alimentos, entre otros. Se suma la puntuación obtenida en los ítems 3, 1, 2, 20, 12, $6,15,17$ y 4 . Por último, el tercer factor se ha denominado "Observación de Impulsividad", ya que han saturado en mayor medida aquellos ítems relativos a consumo de sustancias, pensamientos/intentos de suicidio y agresividad. Se debe sumar la puntuación obtenida en los ítems 23, 26, 22, 24, 25 y 27. Por último, para hallar la puntuación total de la subescala de Síntomas del COBU de 25 items, se suman las puntuaciones obtenidas en los tres factores. La puntuación fluctúa entre 0 y 50 puntos.

\section{Fiabilidad e intercorrelaciones}

El coeficiente alfa de Cronbach obtenido para el Factor 1 alcanza el valor de 0.77 , para el Factor 2 tiene un valor de 0.76 y para el Factor 3 tiene un valor de 0.62 (ver Tabla 4). La fiabilidad del instrumento total COBU es de 0.81. Los resultados de las correlaciones entre los diferentes factores del COBU (ver Tabla 5), indican que los factores que más correlacionan entre sí son la Observación de la Sobreingesta (Factor 1) y la Observación de Periodos Restrictivos (Factor 2), cuyo valor de correlación es moderado (rho $=0.39 ; p<0.01$ ) El resto de co- rrelaciones son bajas, lo que significa que cada factor mide un constructo diferente.

\section{Validez convergente}

El análisis de correlación con otros instrumentos para examinar la validez convergente se ha realizado con distintas submuestras clínicas (ver Tabla 5). Respecto al cuestionario contestado por los cuidadores, el ABOS (instrumento de observación de la conducta anoréxica) ha mostrado correlaciones positiva y elevadas de forma estadísticamente significativas con el COBU total $($ rho $=$ $0.58 ; p<0.01$ ). Al estudiar la correlación de la escala ABOS con cada subescala del COBU, podemos observar que correlaciona de forma positiva con todas las subescalas y de forma destacada con la Observación de Periodos de Restricción, mostrando un valor moderadamente elevado (rho $=0.64 ; p<0.01$ ), como cabría esperar.

En cuanto al cuestionario contestado por las pacientes, la correlación del cuestionario BITE (específico de evaluación de sintomatología $\mathrm{BN}$ ) y el COBU total presenta un valor moderado (rho $=0.40 ; p<0.01)$. También se ha correlacionado el COBU con las cuatro subescalas relacionadas con la patología alimentaria e impulsividad del EDI-2. Los resultados indican que la Observación de Sobreingesta correlaciona significativamente con la subescala Bulimia EDI-2 (rho $=0.46 ; p<0.01$ ), con la subescala de Impulsivida EDI-2 (rho $=0.37 ; p<0.01)$ y con la subescala Insatisfacción Corporal EDI-2 (rho $=0.30 ; p<$ 0.01). Por otro lado, destaca que la Observación de Periodos de Restricción correlaciona moderada y significativamente con la subescala Obsesión por la delgadez (rho = $0.42, p<0.01$ ), y baja con las otras tres subescalas del EDI-2. Y la subescala de Impulsividad del cuestionario COBU se asocia moderada y significativamente con la subescala Impulsividad EDI-2 ( $(\mathrm{ho}=0.37 ; p<0.01)$.

\section{Validez clínica}

Para examinar la validez clínica, es decir, para saber si el Cuestionario de Observación de la Conducta Bulí- 
Tabla 4. Análisis Factorial Exploratorio a través del Método de Componentes Principales (CP) y Ejes a Tabla 4. Análisis factorial exploratorio del Cuestionario de la Conducta Bulímica (COBU). Métodos de componentes principales (CP) y ejes principales (EP)

\begin{tabular}{|c|c|c|c|c|c|c|}
\hline & \multicolumn{2}{|c|}{$\begin{array}{c}\text { Factor 1: } \\
\text { Observación } \\
\text { Sobreingesta }\end{array}$} & \multicolumn{2}{|c|}{$\begin{array}{c}\text { Factor 2: } \\
\text { Observación } \\
\text { de Restricción }\end{array}$} & \multicolumn{2}{|c|}{$\begin{array}{c}\text { Factor 3: } \\
\text { Observación } \\
\text { Impulsividad }\end{array}$} \\
\hline & $\mathrm{CP}$ & EP & $\mathrm{CP}$ & EP & $\mathrm{CP}$ & $\mathrm{EP}$ \\
\hline \multicolumn{7}{|l|}{ FACTOR 1: Observación de Sobreingesta $(\alpha=0.77)$} \\
\hline 11 ¿Observa de forma reiterada que desaparece comida en casa? & 0.72 & 0.68 & & & & \\
\hline 14 ¿Tiende a atracarse (ingesta compulsiva de alimentos)? & 0.69 & 0.65 & & & & \\
\hline 13 ¿Tiende a comer más? & 0.65 & 0.57 & & & & \\
\hline 10 ¿Tiene evidencias de que su hija/o come a escondidas? & 0.54 & 0.49 & & & & \\
\hline 19 ¿El trastorno alimentario de su hija/o interfiere en sus actividades cotidianas? & 0.52 & 0.45 & & & & \\
\hline 7 ¿Observa que toma grandes dosis de chicles y/o Coca-Cola y/o café? & 0.50 & 0.41 & & & & \\
\hline 16 ¿Observa que oscila de peso en cortos periodos de tiempo? & 0.49 & 0.40 & & & & \\
\hline $8 \quad$ ¿Ingiere su hija/o la comida con gran rapidez? & 0.45 & 0.36 & & & & \\
\hline 21 ¿Se irrita con facilidad y en ocasiones no maneja su ira? & 0.41 & 0.37 & & & & \\
\hline 18 ¿Considera que la máxima preocupación de su hija/o es la comida? & 0.40 & 0.38 & 0.54 & & & \\
\hline *28 ¿Vomita su hija/o tras un atracón? & 0.30 & 0.25 & & & & \\
\hline \multicolumn{7}{|l|}{ FACTOR 2: Observación de Periodos Restrictivos $(\alpha=0.76)$} \\
\hline 3 ¿Evita comer determinados alimentos que considera "hipercalóricos"? & & & 0.71 & 0.66 & & \\
\hline $1 \quad ¿$ Observa que su hija/o se somete a constantes dietas? & & & 0.63 & 0.55 & & \\
\hline 2 Durante las comidas. ¿come pocas cantidades? & & & 0.58 & 0.50 & & \\
\hline 15 ¿Verbaliza su hija/o autodesprecio. cada vez que se excede comiendo? & & & 0.57 & 0.53 & & \\
\hline $\begin{array}{l}6 \text { ¿Cada vez que su hija/o se salta una dieta que ella misma se impone, se mues- } \\
\text { tra hostil, tensa/o, irascible? }\end{array}$ & & & 0.56 & 0.50 & & \\
\hline $\begin{array}{l}20 \text { ¿Se niega a acudir a determinadas situaciones sociales, como bodas, cum- } \\
\text { pleaños u otras celebraciones? }\end{array}$ & & & 0.55 & 0.46 & & \\
\hline 12 Durante los episodios de atracón, ¿come los alimentos que normalmente evita? & & & 0.52 & 0.47 & & \\
\hline 17 ¿Verbaliza su hija/o el temor a subir de peso y a convertirse en obesa/o? & & & 0.51 & 0.46 & & \\
\hline $4 \quad$ ¿Se salta comidas? & & & 0.43 & 0.36 & & \\
\hline \multicolumn{7}{|l|}{ FACTOR 3: Observación de Impulsividad $(\alpha=0.62)$} \\
\hline 23 ¿Ha observado a su hija/o agredir a otras personas? & & & & & 0.70 & 0.61 \\
\hline 22 ¿Alguna vez ha observado a su hija/o agredirse? & & & & & 0.63 & 0.53 \\
\hline 26 ¿Ha verbalizado su hija/o en alguna ocasión pensamientos de suicidio? & & & & & 0.58 & 0.50 \\
\hline 25 ¿Existen pruebas de que su hija/o consume drogas? & & & & & 0.52 & 0.37 \\
\hline 27 ¿Ha tenido intentos de suicidio? & & & & & 0.47 & 0.34 \\
\hline 24 En los últimos seis meses, ¿ha observado a su hija/o borracha/o en varias ocasiones? & & & & & 0.45 & 0.31 \\
\hline *29 ¿Ha llegado a robar para satisfacer la necesidad de atracarse? & & & & & 0.33 & 0.30 \\
\hline *30 ¿Tiende su hija/o a mantener relaciones sexuales inestables? & & & & & 0.28 & 0.26 \\
\hline Porcentaje de varianza explicada (excluyendo los ítems 28,29 y 30 ) & 14.04 & 11.42 & 14.31 & 11.61 & 8.85 & 5.97 \\
\hline Porcentaje acumulado de la varianza explicada (excluyendo los ítems 28,29 y 30) & 14.04 & 11.42 & 28.35 & 23.03 & 37.20 & 29.00 \\
\hline
\end{tabular}

*Items con saturación <.35 (no se han tenido en cuenta para calcular la varianza total ni el cálculo total de las subescalas). 
Tabla 5. Medias, DT, correlaciones e intercorrelaciones del COBU (rho de Spearman)

\begin{tabular}{|c|c|c|c|c|}
\hline & $\begin{array}{c}\text { Factor 1: Observación } \\
\text { de Sobreingesta }\end{array}$ & $\begin{array}{l}\text { Factor 2: Observación } \\
\text { de Periodos de } \\
\text { Restricción }\end{array}$ & $\begin{array}{l}\text { Factor 3: Observación } \\
\text { de Impulsividad }\end{array}$ & $\begin{array}{c}\text { Cuestionario "COBU" } \\
\text { Total }\end{array}$ \\
\hline $\begin{array}{l}\text { Padres } \\
\text { ABOS }\end{array}$ & $0.31 * *$ & $0.64 * *$ & $0.22 * *$ & $0.58 * *$ \\
\hline $\begin{array}{l}\text { Pacientes } \\
\text { BITE }\end{array}$ & $0.39 * *$ & $0.24 * *$ & $0.17^{*}$ & $0.40 * *$ \\
\hline $\begin{array}{c}\text { EDI-2 } \\
\text { "Obsesión por la } \\
\text { delgadez" }\end{array}$ & $0.26^{* *}$ & $0.42 * *$ & 0.03 & $0.37 * *$ \\
\hline $\begin{array}{l}\text { EDI-2 } \\
\text { "Bulimia" }\end{array}$ & $0.46^{* *}$ & $0.17^{*}$ & 0.15 & $0.39 * *$ \\
\hline $\begin{array}{c}\text { EDI-2 } \\
\text { "Insatisfacción corpo- } \\
\text { ral" }\end{array}$ & $0.30 * *$ & $0.18^{*}$ & $0.16^{*}$ & $0.28 * *$ \\
\hline $\begin{array}{c}\text { EDI-2 } \\
\text { "Impulsividad" }\end{array}$ & $0.36^{* *}$ & $0.18^{*}$ & $0.37 * *$ & $0.37 * *$ \\
\hline $\begin{array}{l}\text { Factor 1: } \\
\text { Sobreingesta }\end{array}$ & - & $0.39 * *$ & $0.21 * *$ & $0.84 * *$ \\
\hline $\begin{array}{l}\text { Factor 2: } \\
\text { Restricción }\end{array}$ & - & - & 0.13 & $0.77 * *$ \\
\hline Factor 3: Impulsividad & - & - & - & $0.38^{* *}$ \\
\hline Media $(D T)$ & $\begin{array}{c}11.6(5.4) \\
\text { Rango }=0-20\end{array}$ & $\begin{array}{c}8.7(5.07) \\
\text { Rango }=0-18\end{array}$ & $\begin{array}{c}2.01(2.4) \\
\text { Rango }=0-12\end{array}$ & $\begin{array}{c}23.3(9.8) \\
\text { Rango }=0-50\end{array}$ \\
\hline
\end{tabular}

Nota $: \mathrm{ABOS}=$ Escala de Observación de la Conducta Restrictiva; BITE = Test de Bulimia de Edimburgo; EDI-2 = Inventario de Trastornos Alimentarios. $* p<.05, * * p<.01$.

mica (COBU) discrimina entre los diferentes diagnósticos, se realizó un ANOVA de un factor. La Tabla 6 muestra los resultados, donde el primer factor (Sobreingesta) hay diferencias de medias entre los grupos (AN, BN y TA), donde los cuidadores observan menos periodos de sobreingesta en pacientes con AN comparados con $\mathrm{BN}$ y TA, que son obtienen resultados similares. En cuanto al segundo factor (Restricción) solamente difieren las medias entre $\mathrm{BN}$ y TA; mientras que para el tercer factor (Impulsividad) los grupos no difieren en sus medias ( $p>$ $0,01)$, y no logra discriminar entre pacientes. En las pruebas post hoc se utilizó el procedimiento de Bonferroni, y los resultados indican que tanto para el primer factor como para el segundo existen diferencias significativas $(p<0.05)$.

\section{Discusión}

El objetivo principal de este trabajo ha sido desarrollar y validar un instrumento aplicado a cuidadores para observar la conducta y actitudes en pacientes con bulimia nerviosa, denominado COBU (Cuestionario de Ob- servación de la Conducta Bulímica). Los resultados tras los análisis factoriales exploratorios sugieren tres factores, las cuáles pueden ser evaluadas por 3 subescalas: Observación de Sobreingesta (evalúa la desaparición de comida en casa, episodios de atracones, irritabilidad, entre otros), la Observación de Periodos de Restricción (evalúa la evitación de alimentos, el sometimiento a dietas, ingerir pocas cantidades de comida, entre otras), y por último, la Observación de Impulsividad (evalúa el consumo de sustancias, la agresividad extrema, entre otras). Estas tres subescalas conformaría la escala de Síntomas del cuestionario COBU, más una escala de Gravedad, que consta de 2 ítems relacionados con la frecuencia de conductas compensatorias y episodios de atracones. En resumen, tantos los datos basados en componentes principales como en ejes principales, han obtenido una misma estructura factorial en una muestra de 162 cuidadores, y la varianza explicada se reduce de $37.3 \%$ a un $29 \%$, respectivamente, para los mismos 25 ítems. Esta estructura factorial es muy similar a la obtenida por Vandereycken et al., (1992) para el cuestionario ABOS, que también cuenta con tres subescalas diferen- 
Tabla 6. Diferencias en las subescalas del COBU entre los distintos grupos diagnósticos

\begin{tabular}{|c|c|c|c|c|}
\hline & $\begin{array}{c}\mathrm{AN} \\
n=26\end{array}$ & $\begin{array}{c}\mathrm{BN} \\
n=126\end{array}$ & $\begin{array}{c}\text { TA } \\
n=10\end{array}$ & \\
\hline Subescala COBU & $M(D T)$ & $M(D T)$ & $M(D T)$ & $F$ \\
\hline Observación de Sobreingesta & $7.0^{\mathrm{a}}(4.3)$ & $12.5^{\mathrm{b}}(5.0)$ & $11.6^{\mathrm{b}}(5.3)$ & $13.5 * * *$ \\
\hline Observación de Periodos de Restricción & $7.3^{\mathrm{a}, \mathrm{b}}(5.1)$ & $9.2^{\mathrm{a}}(5.0)$ & $4.7^{\mathrm{b}}(3.5)$ & $4.7 * *$ \\
\hline Observación de Impulsividad & $1.5(1.9)$ & $2.1(2.5)$ & $1.5(2.4)$ & 0.9 \\
\hline
\end{tabular}

Nota. Las medias que comparten superíndice no son significativamente diferentes entre sí (prueba de Bonferroni, $p<.05$ ). AN = anorexia nerviosa, $\mathrm{BN}=$ bulimia nerviosa; $\mathrm{TA}=$ trastorno de atracón. ${ }^{* *} p<.01,{ }^{* * *} p<.001$.

ciadas con el fin de observar la psicopatologia en anorexia nerviosa. Destaca el Ítem 18 del COBU “¿Considera que la máxima preocupación de su hija/o es la comida?", que satura en dos factores, y que puede tener sentido clínico que esta preocupación ocurra a la paciente tanto en periodos de sobreingesta como en periodos de restricción. Finalmente se decidió no eliminarlo y que formara parte de la Observación de la Sobreingesta, ya que es una preocupación que refieren las pacientes continuamente.

Los resultados también proporcionan evidencia sobre otras propiedades psicométricas, como la fiabilidad y la validez convergente, medidas a través de cuestionarios en cuidadores y en las propias pacientes. Respecto al análisis de la consistencia interna, mostró ser satisfactoria en la homogeneidad entre todos los ítems del cuestionario COBU total. Los coeficientes alfa de Cronbach para las dos subescalas encontradas fueron mayor 0.70 (sugerirían que existe una adecuada consistencia interna). La subescala de Impulsividad obtiene una fiabilidad menor de 0.62 , esto puede deberse a que la mayoría de las respuestas de los cuidadores a estos ítems era 0 , es decir, que había pocas adolescentes que presentaban estas conductas de riesgo. De forma similar el ABOS en su muestra original obtiene un índice de Cronbach de 0.80, 0.69, and 0.69 respectivamente para las subescalas Preocupación por peso y comida, Negación de los problemas e Hiperactividad (Vandereycken et al.,1992). Los autores refieren que obtienen una fiabilidad suficiente y explican en el caso de la escala de Hiperactividad en AN, que de forma controvertida las pacientes según van reduciendo el peso, presentan una mayor actividad física de forma constante, y es importante que los cuidadores observen este síntoma, ya que señala una mayor severidad de la patología en términos clínicos, y no una respuesta común de las adolescentes. Por lo tanto, hemos decidido no eliminar esta subescala con menor consistencia interna, porque medir el perfil de impulsividad en BN es muy importante para marcar la severidad clínica de la paciente desde el inicio; que los cuidadores sean observadores del potencial riesgo de conductas autolesivas o en relación a consumo de sustancias, proporciona claramente, un informe más fiable en la evaluación inicial de las pacientes como de su proceso de recuperación, como así recomienda la Guía de la APA (Yager, Anderson y Devlin, 2000) o la Guía de Stanford (Rome et al., 2003). También se promueve la inclusión de la familia en el tratamiento FBT, tanto en la evaluación como en el tratamiento (Loeb et al., 2012; Eisler et al., 2007; Haigh y Treasure, 2003).

Como se esperaba, se encontró una asociación alta significativamente con la escala ABOS de padres, indicando que se evalúan constructos muy similares, es decir, la observación externa de síntomas de patología alimentaria, relacionada con los ciclos de sobreingesta y restricción de comida principalmente. Respecto a la validez convergente con los dos cuestionarios de las propias pacientes, se esperaba que fueran entorno al 0.50 , sin embargo, han sido correlaciones moderadas, aunque las asociaciones más alta entre subescalas tienen un sentido clínico adecuado. Como se observa en la subescala de Observación de la Restricción se asocia de forma más elevada con Obsesión de la delgadez del EDI-2, que tiene ítems de deseo de adelgazar o de conductas de dieta. Mientras la subescala de Sobreingesta se asocia a la subescala de Bulimia del EDI-2 que evalúa el periodo de voracidad presentado las pacientes con BN (Garner, 1998). Otra hipótesis explicativa a los resultados de encontrar una menor asociación de la esperada, puede tener que ver con la dificultad en la percepción de las propias pacientes de su enfermedad (Rome et al., 2003), una parte de las pacientes con patología alimentaria niegan su sintomatología y más al inicio del tratamiento, estando más del $60 \%$ de nuestra muestra en la evaluación inicial. En esta línea, algunos autores comparan la entrevista realizada al niño o adolescente y los cuestionarios completados por padres, y no siempre concuerda el contexto familiar y/o las conductas y/o los tiempos de los sucesos entre ambos (Tanofsky-Kraff, Yanovski, y Yanovski, 2005). Por tanto, es relevante la inclusión siempre que se pueda de los cuidadores de las pacientes, cuanto más informadores sobre la enfermedad, más fiable es el diagnóstico y el pronóstico establecido (Yager, Anderson y Devlin, 2000). 
El cuestionario COBU discrimina de forma clínica las observaciones de la patología bulímica, y no registra de igual modo la patología restrictiva y de control extremo del peso, típica de las pacientes con AN. Por eso motivo, las puntuaciones en padres de pacientes con AN es bastante menor, para la Observación de la Sobreingesta y las conductas compensatorias. Es llamativo que no haya diferencias en la subescala de Observación de la Impulsividad, y esto se podría referir a que hay que mejorar los ítems de este factor, otra hipótesis explicativa es que al ser una prevalencia de casos pequeña que presentan este perfil en la muestra, no se puede obtener una adecuada fiabilidad. Se recomienda utilizar en otra poblaciones de pacientes con $\mathrm{BN}$ adultas jóvenes o mayores, para ver cómo funciona estos ítems cuando el curso de la psicopatología y la severidad se van agravando en las pacientes. Al separar las pacientes con TA según DSM-5 (APA, 2013), se han quedado en una muestra muy pequeña, y sin embargo, las puntuaciones medias en las subescalas del COBU apuntan a que presentan unos patrones diferenciadores. Sería importante seguir investigando en una muestra de un tamaño mayor de pacientes con TA, para valorar cómo funciona la impulsividad, ya que cabría esperar que fuera una característica diferenciadora entre ambos diagnóstico.

\section{Limitaciones}

Una de las limitaciones que cabe destacar es el formato de respuesta escogido para el cuestionario. El hecho de que una de las opciones de respuesta sea que el observador no tenga la certeza de tener esa información (?) sesga los resultados, ya que concede 1 punto a la suma total por cada respuesta dada por los cuidadores de los pacientes. Debido a la fiabilidad menor de la subescala de Impulsividad del COBU aplicada a padres, que también obtiene una fiabilidad justa la propia subescala de Impulsividad del EDI-2 en pacientes, puede finalmente ir relacionado con una baja prevalencia de estas conductas en la muestra. Por otra parte, sería recomendable ampliar la muestra de pacientes adultas con BN como también utilizar otro tipo de cuestionarios para los cuidadores, que evalúen constructos como ansiedad, depresión o emoción expresada, entre otros, para obtener una visión más global de la percepción de los padres, ya que los cuidadores pueden sesgar las respuestas en el cuestionario debido a toda la carga emocional que presentan (Sepúlveda et al., 2008; 2009; Cole y Kazarian, 1988) y que les impide, en cierta medida, poder observar la sintomatología bulímica que presentan las pacientes con este trastorno alimentario. Como también sería importante una medida test-rest para examinar la estabilidad temporal del cuestionario desa- rrollado. Como también observar las diferencias entre padres y madres en el uso del COBU, y examinar la fiabilidad inter-jueces entre ambos. La muestra actual de cuidadores sólo contaba con un 7\% de padres, sin embargo, los resultados en otros cuestionarios recientemente validados en TCA, indican que existen importantes diferencias en cómo perciben la enfermedad, y por tanto, en las respuesta que ofrecen a su entorno familiar según género (Sepúlveda et al., 2014) o según seas cuidador principal o secundario (Berbel et al., 2010). Para futuras replicaciones, sería recomendable seguir las siguientes indicaciones: utilizar una mayor muestra para realizar los estudios de validez convergente, concurrente, test-rest, y cambiar el formato de respuesta de los ítems a un formato de tipo Likert, para evitar sesgos en los resultados.

\section{Referencias}

American Psychiatric Association (2000). Diagnostic and statistical manual of mental disorders: DSM-IV-TR (4th Ed.). Washington, DC: APA.

American Psychiatric Association (2013). Diagnostic and Statistical Manual for Mental Disorders (5th Ed.). Arlington, V.A.: APA.

Berbel, E., Sepúlveda, A.R., Greaell, M., Andrés, P., Carrobles, J., y Morande, G. (2010). Valoración del estado de salud y psicopatología de los familiares en el trastorno del comportamiento alimentario: Diferencias entre cuidadores principales y secundarios. Revista de Psicopatología Clínica, 5,179-192.

Carral-Fernández, L., Sepúlveda, A. R., Gómez Del Barrio, A., Graell, M. y Treasure, J. (2013). The Spanish validation of an Eating Disorders Symptom Impact Scale (EDSIS) among caregivers. Psychiatry Research, 209, 626-31

Castro, U., Larroy, C. y Gómez, M. A. (2010). Intervención cognitivo conductual para pacientes adolescentes y sus padres en el tratamiento de la bulimia nerviosa. Revista de Psicopatología y Psicología Clínica, 15, 49-60.

Cohen, J. (1988). Statistical power analysis for the behavioral sciences. 2nd edition. Hillsdale, NJ: Larence Earlbaum Associates.

Cole, J. D. y Kazarian, S. S. (1988). The Level of Expressed Emotion Scale: A new measure of expressed emotion. Journal of Clinical Psychology, 44, 392-397.

Eisler, I., Dare, C., Hodes, M., Russell, G., Dodge, E. y Le Grange, D. (2000). Family therapy for adolescent anorexia nervosa. The results of a controlled comparison of two family interventions. Journal of Child Psychology and Psychiatry and Allied Disciplines, 41, 727-736.

Eisler, I., Dare, C., Russell, G.F.M., Szmukler, G., Le Grange, D. y Dodge, E. (1997). Family and individual therapy in anorexia nervosa: A five-year follow-up. Archives of General Psychiatry, 54, 1025-1030.

Eisler, I., Simic, M., Russell, G.F.M. y Dare, C. (2007). A randomised controlled treatment trial of two forms of family therapy in adolescent anorexia nervosa: A five-year follow-up. Journal of Child Psychology and Psychiatry, 48, 552-560 
Fairburn, C.G., Cooper, Z. y O'Connor, M. (2008). Eating disorder examination, edition 16.0. En: C.G. Fairburn. Cognitive behavior therapy and eating disorders. New York: Guilford Press.

Fairburn, C.G., Cooper, Z. y Shafran, R. (2003). Cognitive behaviour therapy for eating disorders: a "transdiagnostic" theory and treatment. Behaviour Research and Therapy, 41, 509-528. Garner, D.M. (1991). Eating Disorder Inventory-2: Professional Manual. Odesa: Psychological Assessment Resources.

Garner, D.M. (1991). Eating disorder inventory-2: Professional Manual. Odesa: Psychological Assessment Resources.

Garner, DM. (1998). EDI-2. Inventario de trastornos de la conducta alimentaria. Madrid: TEA Ediciones.

Grupo de trabajo de la Guía de Práctica Clínica sobre TCA (2009). Guía de práctica clínica sobre trastornos de la conducta alimentaria. Barcelona: Agència d'Avaluació de Tecnologia i Recerca Mèdiques de Cataluña.

Haigh, R. y Treasure, J. (2003). Investigating the needs of carers in the area of eating disorders: Development of the Carers' Needs Assessment Measure (CaNAM). European Eating Disorders Review, 11, 125-141.

Henderson, M. y Freeman, L. (1987) A self-rating scale for bulimia (The "BITE"). British Journal of Psychiatry, 150, 18-24.

Johnson, W. G., Grieve, F.G., Adams, C.D., y Sandy, J. (1999). Measuring binge eating in adolescent: Adolescent and parent versions of the questionnaire of eating and weight patterns. International Journal of Eating Disorders, 26, 301-314.

Le Grange, D., Crosby, R.D., Rathouz, P.J. y Leventhal, B.L. (2007). A Randomized controlled comparison of Family-Based Treatment and supportive psychotherapy for adolescent bulimia nervosa. Archives of General Psychiatry, 64, 10491056.

Le Grange, D. y Lock, J. (2007). Treating bulimia in adolescence: A family-based approach. New York: Guilford Press.

Lock, J., Agras, W.S, Bryson, S. y Kraemer H.C. (2005). A comparison of short- and long-term Family Therapy for adolescent anorexia nervosa. Journal of the American Academy of Child \& Adolescent Psychiatry, 44, 632-639.

Lock, J., Le Grange, D., Agras, W.S y Dare C. (2001). Treatment manual for anorexia nervosa: A family-based approach. New York: Guilford Press.

Loeb, K. L. (2007). Eating Disorder Examination QuestionnaireParent Version (P-EDEQ), Version 1.4. Unpublished measure based on Fairburn, C.G. y Beglin, S. (2008). Eating Disorder Examination Questionnaire (EDE-Q). En: C.G. Fairburn (Ed.). Cognitive Behavior Therapy and Eating Disorders (pp. 265-308). NY.: Guilford Press.

Loeb, K. L. (2008). Eating Disorder Examination-Parent Version (P-EDE). Version 1.4. Unpublished measure based on Fairburn, C.G., Cooper y O'Connor, M. E. (2008). The Eating Disorder Examination (EDE, Edition 16.0). En C.G. Fairburn (Ed.). Cognitive Behavior Therapy and Eating Disorders (pp. 265-308). NY.: Guilford Press.

Loeb, K. L., Lock J., Greif, R. y Le Grange, D. (2012). Transdiagnostic theory and application of family-based treatment for youth with eating disorders. Cognitive and Behavioral Practice, 19, 17-30.

Martin, J., Padierna, A., González, N,. Aguirre, U., Lafuente, I., Muñoz, P., Quintana, J. M. (2013). Evaluation of the psychometric characteristics of the Spanish version of the Anorectic Behavior Observation Scale. International Journal of Clinical Practice, 68, 83-93.

Oliva, L., Gandarillas, A., Sonego, M., Díez-Gañán, L. y Ordobás, M. (2012). Vigilancia epidemiológica de los trastornos del comportamiento alimentario y conductas relacionadas. Comunidad de Madrid, 2011. Boletín Epidemiológico de la Comunidad de Madrid, 18, $\mathrm{N}^{\circ} 8$.

Quiles, Y., Quiles, M.J., Pamies, L., Sepúlveda, A.R. y Treasure, J. (2015). The Spanish validation of the Accommodation and Enabling Scale for Eating Disorders Among Carers: A pilot study. European Eating Disorders Review, 0-00.

Rivas, T., Bersabé, R., y Jiménez, M. (2004). Fiabilidad y validez del Test de Investigación Bulímica de Edinburgo (BITE) en una muestra de adolescentes españoles. Psicología Conductual, 12, 447-461.

Rome, E. S., Ammerman, S., Rosen, D. S., Keller, R. J., Lock, J., Mammel, K. A., \& Silber, T. J. (2003). Children and adolescents with eating disorders: the state of the art. Pediatrics, 111, e98-e108.

Russell, G.F.M., Szmukler, G.I., Dare, C., Eisler, I. (1987). An evaluation of family therapy in anorexia nervosa and bulimia nervosa. Archives of General Psychiatry, 44, 1047-1056.

Sepúlveda, A.R., Anastasiadou, D., Rodriguez, L., Almendros, C., Andrés, P., Vaz, F. y Graell, M. (2014). Spanish validation of the family questionnaire (FQ) in families of patiens with an eating disorder. Psicothema, 26, 321-327.

Sepúlveda, A.R., Whitney, J., Hankins, M. y Treasure J. (2008). Development and validation of an Eating Disorders Symptom Impact Scale (EDSIS) for carers of people with eating disorders. Health Qualitive Life Outcomes, 6, 28.

Sepúlveda, A.R., Kyriacou, O. y Treasure, J. (2009). Development and validation of the Accomodation and Enabling Scale for Eating Disorders. BMC Health Service Research, 9, 177.

Tanofsky-Kraff, M., Yanovski, S.Z. y Yanovski, J.A. (2005). Comparison of child interview and parent reports of children's eating disordered behaviors. Eating Behaviors, 6,1, 95-99.

Toro, J., Guerrero, M., Sentis, J., Castro, J. y Puertolas, C. (2008). Eating disorders in ballet dancing student: Problems and risk factors. European Eating Disorders Review, 17, 40-49.

Vandereycken, W. (1992). Validity and reliability of the anorectic behavior observation scale for parents. Acta Psychiatrica Scandinavica, 85, 163-6.

Wiedemann, G., Rayki, O., Feinstein, E., y Hahlweg, K. (2002). The Family Questionnaire: Development and validation of a new self-report scale for assessing expressed emotion. Psychiatry Research, 109, 265-279.

Yager, J., Anderson, A. y Devlin, M. (2000). American Psychiatric Association Practice Guideline for the Treatment of Patients with Eating Disorders. American Journal of Psychiatry, 157, $1-39$. 
Anexo I. Cuestionario de Observación de la Conducta Bulímica (COBU)

Nombre del paciente:

Fecha:

Rellenado por:

\begin{tabular}{|l|l|}
\hline & Madre \\
\hline & Padre \\
\hline & Otro: $\ldots \ldots \ldots \ldots \ldots \ldots \ldots \ldots \ldots \ldots \ldots \ldots$ \\
\hline
\end{tabular}

Instrucciones: Conteste las siguientes preguntas sobre la base de las observaciones durante los últimos tres meses en casa. Conteste SI o NO, únicamente si está seguro (por ejemplo, si observó lo que sucedía). Conteste (?) si no está seguro (por ejemplo, si no ha podido observarlo, pero lo ha oído o supone que ha sucedido).

1. ¿Observa que su hija/o se somete a constantes dietas? No prescritas por un médico

2. Durante las comidas, ¿come pocas cantidades?

3. ¿Evita comer determinados alimentos que considera "hipercalóricos"?

4. ¿Se salta comidas?

5. ¿Tiene alguna evidencia de que su hija/o utiliza alguno de los siguientes métodos para perder peso? Rodea en cada caso con un círculo según la frecuencia indicada en la tabla que encontrarás a continuación.

\begin{tabular}{|c|c|c|c|c|c|c|c|c|}
\hline & $\begin{array}{l}\tilde{J} \\
\Xi \\
Z\end{array}$ & 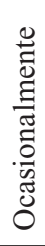 & 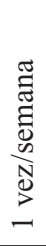 & 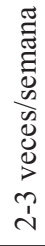 & 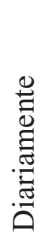 & 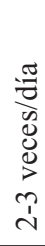 & 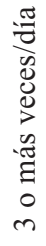 & $\begin{array}{l}\text { D } \\
\text { ే } \\
\text { D } \\
0 \\
\text { Z }\end{array}$ \\
\hline Píldoras adelgazantes & 0 & 2 & 3 & 4 & 5 & 6 & 7 & 1 \\
\hline Diuréticos & 0 & 2 & 3 & 4 & 5 & 6 & 7 & 1 \\
\hline Laxantes & 0 & 2 & 3 & 4 & 5 & 6 & 7 & 1 \\
\hline Vómito autoprovocado & 0 & 2 & 3 & 4 & 5 & 6 & 7 & 1 \\
\hline Ejercicio compulsivo & 0 & 2 & 3 & 4 & 5 & 6 & 7 & 1 \\
\hline
\end{tabular}

6. ¿Cada vez que su hija/o se salta una dieta que ella misma se impone, se muestra hostil, tensa/o, irascible?

7. ¿Observa que toma grandes dosis de chicles y/o Coca-Cola y/o café?

8. ¿Ingiere su hija/o la comida con gran rapidez?

9. ¿Con qué frecuencia cree que se hija/o tiene episodios de atracones? (Por ejemplo, toma una gran cantidad de comida en un intervalo corto de tiempo).

\begin{tabular}{|l|l|l|}
\hline SI & NO & $?$ \\
\hline & & \\
\hline & & \\
\hline & & \\
\hline & & \\
\hline
\end{tabular}

\begin{tabular}{|c|c|c|c|c|c|c|c|}
\hline $\begin{array}{l}\stackrel{\widetilde{J}}{\Xi} \\
Z\end{array}$ & 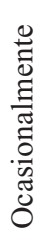 & 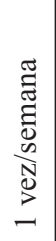 & 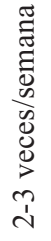 & 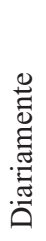 & 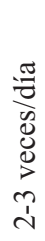 & 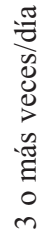 & $\begin{array}{l}\text { D } \\
\text { तु } \\
\text { م } \\
0 \\
Z\end{array}$ \\
\hline 0 & 2 & 3 & 4 & 5 & 6 & 7 & 1 \\
\hline
\end{tabular}

\begin{tabular}{|l|l|l|}
\hline SI & NO & $?$ \\
\hline & & \\
\hline & & \\
\hline & & \\
\hline
\end{tabular}


10. ¿Tiene evidencias de que su hija/o come a escondidas?

11. ¿Observa de forma reiterada que desaparece comida en casa? (En la nevera, despensa...)

12. Durante los episodios de atracón, ¿come los alimentos que normalmente evita?

\begin{tabular}{|l|l|l|}
\hline SI & NO & $?$ \\
\hline & & \\
\hline & & \\
\hline & & \\
\hline
\end{tabular}

\section{Cuando su hija/o se encuentra nerviosa/o (por ejemplo: ante discusiones, exámenes...)}

13. ¿Tiende a comer más?

14. ¿Tiende a tener atracones?

15. ¿Verbaliza su hija/o autodesprecio, cada vez que se excede comiendo?

16. ¿Observa que oscila de peso en cortos periodos de tiempo? (Por ejemplo: a través de la ropa, hinchazón de cara...)

17. ¿Verbaliza su hija/o el temor a subir de peso y a convertirse en obesa/o?

18. ¿Considera que la máxima preocupación de su hija/o es la comida? (Por ejemplo: muchas de sus conversaciones son respecto a la comida, cuerpo, dietas...)

19. ¿El trastorno alimentario de su hija/o interfiere en sus actividades cotidianas? (Por ejemplo: estudiar, salir con amigos...)

20. ¿Se niega a acudir a determinadas situaciones sociales, como bodas, cumpleaños u otras celebraciones?

21. ¿Se irrita con facilidad y en ocasiones no maneja su ira?

22. ¿Alguna vez ha observado a su hija/o agredirse?

23. ¿Y agredir a otras personas?

24. En los últimos seis meses, ¿ha observado a su hija/o borracha/o en varias ocasiones?

25. ¿Existen pruebas de que su hija/o consume drogas? (Por ejemplo: se lo ha contado ella/él misma/o u otras personas, ha encontrado alguna sustancia en casa...)

26. ¿Ha verbalizado su hija/o en alguna ocasión pensamientos de suicidio?

27. ¿Ha tenido intentos de suicidio?

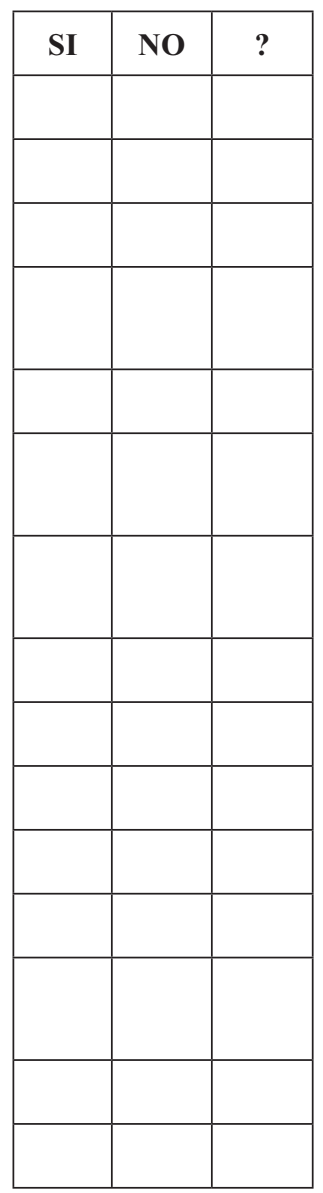

